\title{
Relationship between paravascular abnormalities and foveoschisis in highly myopic patients
}

${ }^{1}$ Department of ophthalmology, Centro de Investigaciones Médico-Sanitarias, Málaga, Spain

${ }^{2}$ Department of ophthalmology, Hospital Virgen de la Victoria de Málaga, Málaga, Spain

${ }^{3}$ Department of Biostatistics, University of Malaga, Málaga, Spain

Correspondence:

R Kamal-Salah, Department of ophthalmology,

Centro de Investigaciones Médico-Sanitarias, Campus teatinos $s / n$ Malaga 29010, Spain Tel: +34 657899932 E-mail: dra.rkamal@ gmail.com

Received: 19 May 2014 Accepted: 23 September 2014

Published online: 7

November 2014

\begin{abstract}
Purpose To describe the prevalence of paravascular abnormalities in highly myopic patients and its relationship with myopic foveoschisis (MF).

Methods Cross-sectional study of 250 highly myopic eyes. All of the patients underwent a complete ophthalmologic examination that included optical coherence tomography . Results Optical coherence tomography images showed 170 eyes (68\%) with paravascular microfolds (PM), 121 eyes (48.4\%) presented paravascular retinal cysts (PC), and 35 eyes (14\%) with paravascular lamellar holes. All the eyes with PCs had PMs. Out of the 250 eyes, $48(19.2 \%)$ had paravascular retinoschisis (PR). All the eyes $(\mathbf{1 0 0 \% )}$ with PR had paravascular cysts and PMs. Sixteen eyes $(6.4 \%)$ had foveoschis. The spherical equivalent $(P<0.00)$, PR $(P=0.01)$, and the presence of tractional structures $(P<0.00)$ were associated with increased risk for foveoschsis in the multivariate study. Conclusions PMs were the lesions most often observed in the paravascular area in highly myopic eyes. MF would be a result of the action of different forces (intra- and extra-ocular forces), specially tractional structures, on precursor lesions (paravascular cyst and paravascular restinoschisis). Further studies are needed to confirm these results. Eye (2015) 29, 280-285; doi:10.1038/eye.2014.255; published online 7 November 2014
\end{abstract}

\section{Introduction}

Myopic foveoschisis (MF) is one of the multiple complications with visual impairment in high
R Kamal-Salah ${ }^{1}$, MJ Morillo-Sanchez ${ }^{2}$, F Rius-Diaz ${ }^{3}$ and JM Garcia-Campos ${ }^{1,2}$ myopia. It was first described by Takano and $\mathrm{Kishi}^{1}$ as a separation of the neurosensory retina into two or more layers in the foveal area. Axial length (AL), macular chorioretinal atrophy, vitreoretinal interface factors and posterior staphyloma had been proposed as factors that contribute to the development of $\mathrm{MF}^{2-8}$ However the pathogenesis has not been clearly established.

The paravascular abnormalities are lesions detected by optical coherence tomography (OCT) around the retinal vessels in highly myopic eyes. Four types of abnormalities have been reported: paravascular retinal cysts (PCs) (identified by OCT as cystoids spaces within the inner retina) ${ }^{9}$ vascular and paravascular microfolds (PMs) (described as a projection of retinal vessels toward the vitreous cavity), 10 paravascular lamellar holes (cracks that extend from the level of the internal limitating membrane to approximately one half of the thickness of the neural retina) and paravascular retinoschisis (PR) (a separation of the retinal layers around the vessels). ${ }^{8}$ These lesions are not frequently diagnosed because it does not affect the visual acuity and it is difficult to detect by funduscopy.

The purpose of this study is to describe the prevalence of paravascular abnormalities in highly myopic patients and its relationship with MF.

\section{Patients and methods}

Two hundred and fifty highly myopic eyes (134 patients) were recruited from the department of ophthalmology 'Virgen de la Victoria' hospital in Malaga (Spain), between 
January 2010 and December 2012. This study was approved by the hospital's institutional review board and was performed in accordance with the World Medical Association's Declaration of Helsinki. Informed consent was obtained for each subject before enrollment. Inclusion criteria was the diagnosis of high myopia, defined as a refractive error (spherical equivalent-SE) of less than -6 diopters (D) or an AL $>26 \mathrm{~mm} .{ }^{11}$ Exclusion criteria included persons under 18 years of age, systemic diseases, previous ocular surgery, and ocular diseases except those resulting from high myopia.

All patients underwent a standard ophthalmologic examination including automated refraction, followed by objective refraction undertaken by a trained optometrist, best corrected snellen visual acuity, intraocular pressure and the AL measurement, anterior segment biomicroscopy, dilated fundus examination by indirect ophthalmoscopy, and slit-lamp examination of the posterior pole using a $+78 \mathrm{D}$ lens. OCT was performed on both eyes of each subject. The refractive error was defined by the SE (SE, defined as the sum of spherical power and half-cylinder power in diopters). The visual acuity was defined as the best corrected visual acuity. The AL was measured with IOLMaster 500 (software version 7.5, Carl Zeiss Meditec AG, Jena, Germany), five measurements were obtained and the mean value was calculated for further statistical analysis. OCT images were obtained with maximal pupil dilatation after ophthalmic evaluation.

The OCT examination was performed using the version Cirrus HD-OCT model 4000 (software version 6.5, Carl Zeiss Meditec). Two types of protocols were used to examine the retina: (a) Macular Cube $512 \times 128$ scan (with 128 horizontal raster, 512A-scans per line, in a $6 \times 6 \mathrm{~mm}$ area): to study the macular area (morphology and central foveal thickness), (b) 5 Line Raster-High definition image analysis (five lines of $6 \mathrm{~mm}$ length, 4096A-scan per line): specially to identify the abnormalities present in the retina around the vascular structures. Scans were performed across the entire extent of the posterior vascular arcade including de macular area. OCT was performed by a single experienced technician. An external fixation was used, when fixation was problematic, the scan was repeated until good fixation was obtained. Only OCT images with signal strengths of more than six out of ten were accepted for analysis.

One of the authors, who was masked to the refractive error, examined carefully the OCT images for the presence of vascular and paravascular retinal microfolds, paravascular cysts, paravascular retinal holes (Figure 1), PR and foveoschisis (Figure 2). We also analyzed the presence of tractional structures.
Statistical analysis was performed using SPSS software for windows (v 15.0, SPSS Inc., Chicago, IL, USA). Data were analyzed using $\chi^{2}$-test and Student's T-test for the univariate analysis of the different factors related to MF. Level of statistical significance was set at $P<0.05$. To determine the factors significantly influencing on $\mathrm{MF}$, multivariate regression analysis was carried out.

\section{Results}

Two hundred and fifty eyes from 134 patients were recruited in this study. Thirty-five patients $(26.11 \%)$ were male and 99 (73.89\%) were female. The mean age was 51.78 years (range 18-88 years). The mean SE was $13.89 \mathrm{D}$ (range from -3 to $-28 \mathrm{D}$ ) and the mean AL was $29.46 \mathrm{~mm}$ (range $24.75-39.29 \mathrm{~mm}$ ). The mean best corrected visual acuity was 0.49 (decimal scale, range from 0.05 to 1.00). The mean intraocular pressure was $15.46 \mathrm{~mm} \mathrm{Hg}$ (range 10-21 mm Hg). We observed nine eyes with $\mathrm{SE}>-6 \mathrm{D}$ but with $\mathrm{AL}>26 \mathrm{~mm}$ and 12 eyes with $\mathrm{AL}<26 \mathrm{~mm}$ but with $\mathrm{SE}<-6 \mathrm{D}$. The characteristics of these patients with or without FV are summarized in Table 1.

The examination of the OCT images showed 170 eyes (68\%) with PMs, 121 eyes (48.4\%) presented PC, and 35 eyes $(14 \%)$ with paravascular lamellar holes. All the eyes with PCs had PMs. Out of the 250 eyes, 48 (19.2\%) had PR. All the eyes (100\%) with PR had paravascular cysts and PMs. Sixteen eyes $(6.4 \%)$ had foveoschis.

In the univariate analysis, Table 2 shows the variables associated with myopic foveschisis: age, SE, PMs, paravascular cysts, retinoschisis without macular involvement, the presence of tractional structures (these include epiretinal membrane, posterior vitreous schisis, vitreomacular traction, and internal limitating membrane detachment), and the presence of epiretinal membrane. There was a significant association with age over 45 years $(P=0.01)$, lower SE $(P=0.00)$, paravascular cyst $(P=0.00)$, PR $(P<0.00)$, tractional structures $(P=0.00)$, and epiretinal membrane $(P=0.00)$. There was no significant association with PMs $(P=0.24)$.

The variables that were independently associated with a significant higher risk of $\mathrm{FV}$ were included in a multivariate analysis (Table 3$)$. The SE $(P<0.00)$, PR $(P=0.01)$, and the presence of tractional structures $(P<0.00)$ were associated with increased risk for foveoschsis.

\section{Discussion}

In this study, we analyzed using OCT 250 eyes of 134 patients with high myopia looking for the presence of paravascular abnormalities and its relation with MF. OCT scans across the entire posterior vascular arcades 
a

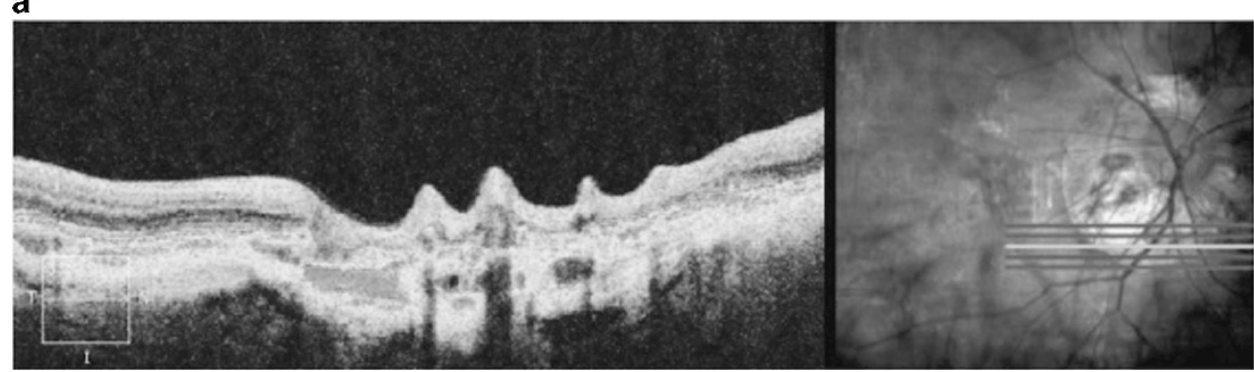

b

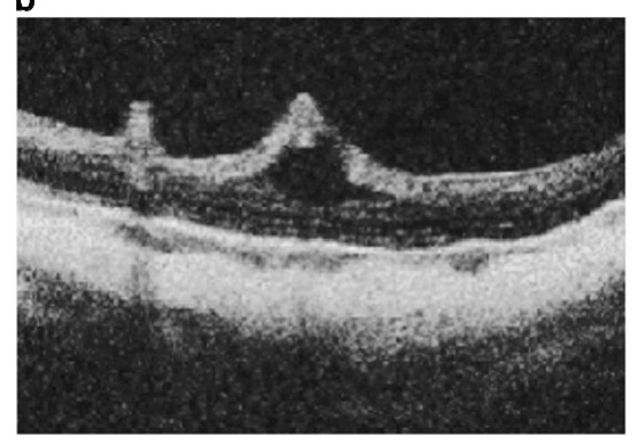

c

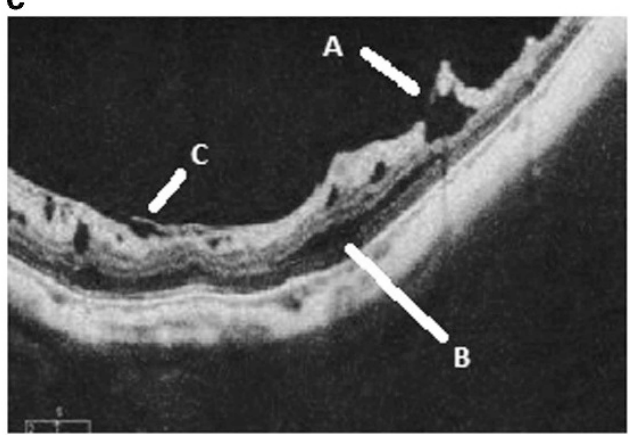

Figure 1 (a) Right eye of a 45-year-old woman. $\mathrm{SE}=-18.5 \mathrm{D}$. AL $=29.1 \mathrm{~mm}$. OCT image (horizontal scan) shows paravascular microfolds as convex projection of the inner retinal layers toward the vitreous cavity. (b) Right eye of a 56-year-old man. SE $=-10.5 \mathrm{D}$. $\mathrm{AL}=27.85 \mathrm{~mm}$. OCT image, horizontal scan, shows paravascular cyst associated with a microfold. The cyst is considered an hyporeflective area between the retinal layers, usually related to microfolds. (c) Right eye of a 58-year-old woman. SE $=-12.5 \mathrm{D}$. $\mathrm{AL}=28.2 \mathrm{~mm}$. OCT image, horizontal scan shows a paravascular retinal hole (A), retinoschisis (B), and tractional internal limiting membrane (C).

showed PMs in $68 \%$ of our eyes. Our results showed that this lesion is the paravascular abnormality more often detected in highly myopic patients. In vitrectomized eyes, PMs had been found in $62 \%$ of highly myopic patients 6 months after surgery. ${ }^{3}$ The prevalence in non vitrectomized highly myopic eyes ranges from 2.9 to $44.6 \%{ }^{6,10}$ We presume that in non vitrectomized eyes, these differences may be partially related to the protocol used in the OCT examination; along the entire length of each posterior arcade vessel or exclusively on the macular area.

PCs were identified in $48.4 \%$ of the highly myopic patients. In a recent study, a prevalence of $49.5 \%$ had been reported. ${ }^{6}$ The pathogenesis of PMs and cysts is still unkown. Vitreoretinal adhesion at the site of the inflexible retinal vessels associated with a generalized expansion of the eyeball in a tangential direction might be the cause of the PMs and the retinal cysts as suggested in different reports. ${ }^{4,5}$ Shimada et $a l^{6}$ detected paravascular cysts in $100 \%$ of the eyes with paravascular retinal microfolds, but only $90.1 \%$ of the eyes with retinal cysts had PMs. These results suggest that the paravascular cysts are the earliest event of mechanical stress on and around the retinal vessels and the formation of a PM facilitate the further splitting of the retinal tissue and cause expansion of the paravascular retinal cyst horizontally and vertically.

In our report, several scans along the entire length of the retinal vessels in temporal arcades, showed that $100 \%$ of the eyes with paravascular cysts had PMs, but only $71.17 \%$ of the eyes with PMs had paravascular cysts. When the two lesions coexist they are located at the same site. The cysts were detected on and around the retinal vessels. Unlike Shimada's report, ${ }^{6}$ we speculate that the first lesion that appears is the PM. Then the action of different forces facilitates the progression to a paravascular cyst.

Examinations detected paravascular retinal holes in 35 of 250 eyes (14\%). All of them were lamellar holes and none full-thickness paravascular retinal holes were found. The paravascular lamellar holes, studied by OCT, were first reported by Shimada, ${ }^{6}$ with a prevalence of $26.8 \%$. This author suggests that in the evolution of paravascular lamellar holes, PCs had to be present as a precursor. It might be a process similar to that of lamellar macular holes. The development of paravascular lamellar holes might be secondary to the rupture of the inner wall of a cyst. ${ }^{6}$

The prevalence of MF was $6.4 \%$. This result is lower than the reported in other studies, that ranges from 


\section{a}

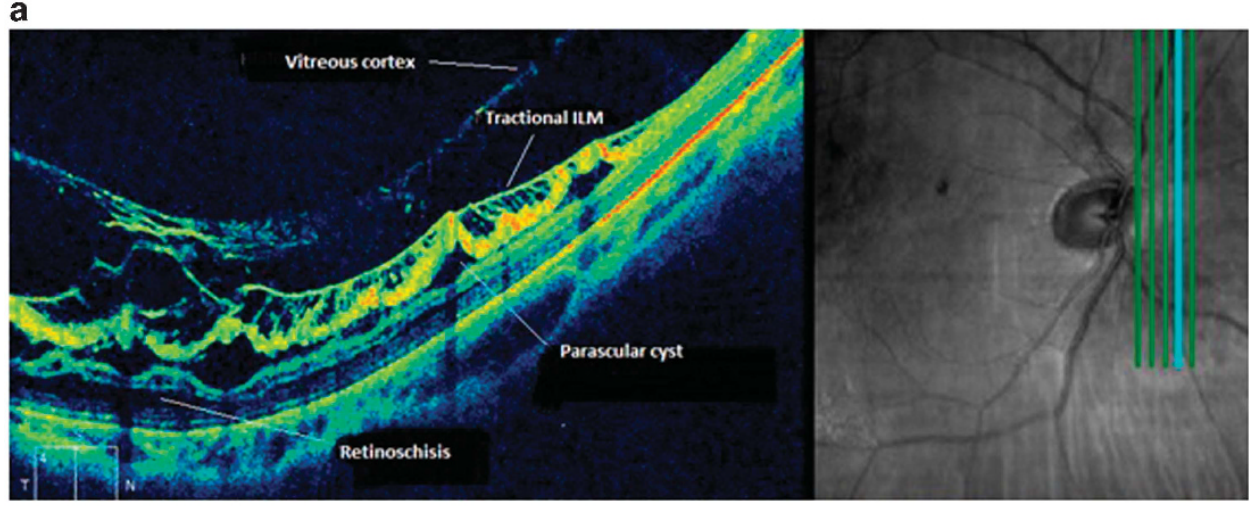

b

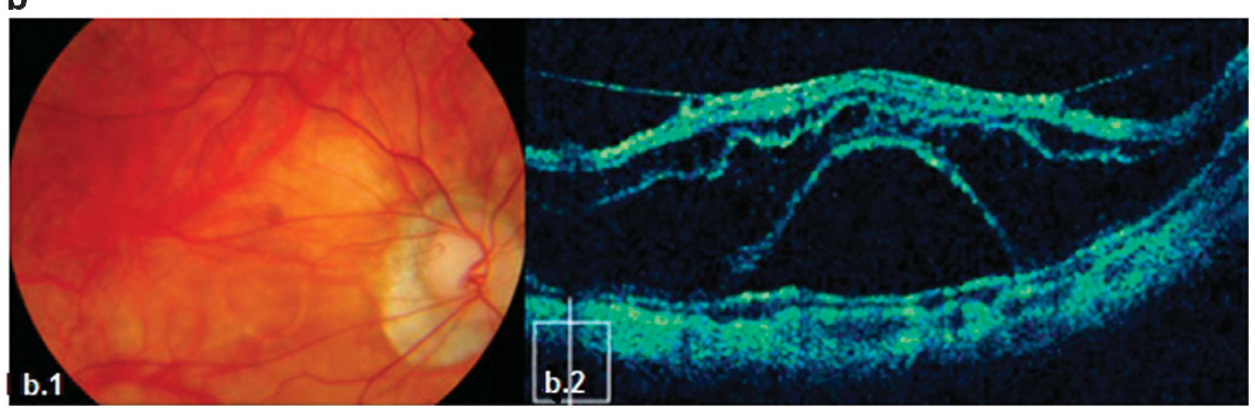

Figure 2 (a) Right eye of a 60-year-old man. $\mathrm{SE}=-18 \mathrm{D} . \mathrm{AL}=29.9 \mathrm{~mm}$. OCT image, vertical scan, shows a case of retinoschisis combined with a tractional syndrome (a thickened posterior hyaloid membrane - vitreous cortex, and a tractional internal limiting membrane). (b) Right eye of a 65-year-old man. $\mathrm{SE}=-21 \mathrm{D}$. $\mathrm{AL}=30.1 \mathrm{~mm}$. (b.1): Color retinography of a myopic fundus showing a posterior staphyloma type I (Curtin classification). (b.2): OCT image, vertical scan, shows a foveal detachment and foveoschisis combined with premacular tractional structures.

Table 1 Clinical characteristics of patients with or without foveoschisis

\begin{tabular}{|c|c|c|c|}
\hline & \multicolumn{2}{|c|}{ Foveoschisis } & \multirow{2}{*}{ P-value } \\
\hline & With $(\mathrm{n}=16)$ & Without $(\mathrm{n}=234)$ & \\
\hline Gender Male (\%) & $2(2.9)$ & $66(67.1)$ & \\
\hline Female $(\%)$ & $14(7.7)$ & $168(92.3)$ & \\
\hline Age (years) mean $( \pm \mathrm{SD})$ & $58.56( \pm 11.26)$ & $51.23( \pm 15.21)$ & $0.05^{\mathrm{a}}$ \\
\hline BCVA (decimal scale) mean ( \pm SD) & $0.28( \pm 0.21)$ & $0.49( \pm 0.33)$ & $0.01^{\mathrm{a}}$ \\
\hline $\mathrm{IOP}(\mathrm{mm} \mathrm{Hg})$ mean $( \pm \mathrm{SD})$ & $16.56( \pm 2.03)$ & $15.39( \pm 2.12)$ & $0.03^{\mathrm{a}}$ \\
\hline Axial length $(\mathrm{mm})$ mean $( \pm \mathrm{SD})$ & $30.21( \pm 3.12)$ & $29.45( \pm 2.29)$ & $0.07^{\mathrm{a}}$ \\
\hline $\mathrm{SE}(\mathrm{D})$ mean $( \pm \mathrm{SD})$ & $-16.26( \pm 7.27)$ & $-13.73( \pm 5.01)$ & $0.05^{\mathrm{a}}$ \\
\hline
\end{tabular}

Abbreviations: BCVA, best corrected visual acuity; D, diopters; IOP, intraocular pressure; N,number of eyes; $\mathrm{SD}$, standard deviation; SE, spherical equivalence.

a Student's T-test.

8 to $34 \%$ depending on the geographic area and the characteristics of the population analyzed. ${ }^{2-4}$ The reason for the difference between other reports and ours might be the fact that our cohort was selected from the general population that visit our clinic and not specifically from the retinal unit.

The pathogenesis of foveoschsis is still unclear. Many authors have studied the relation between the presence of MF and different associated factors. They distinguish intra- and outer-ocular wall factors in the development of
MF. 2,5,9 The outer ocular forces include chorioretinal atrophy, posterior staphyloma, and longer AL. The intraocular forces include epiretinal membrane, vitreomacular traction, residula focal vitreoretinal adhesion, and retinal vessel stiffness. ${ }^{4}$

In the univariate analysis we observed a significant association between the presence of MF and age (over 45 years), SE, paravascular cysts, PR, tractional structures, and epiretinal membrane. These results coincide with other authors. ${ }^{2,9}$ However, it is important to note that we 
Table 2 Univariate analysis of factors associated with myopic foveoschisis

\begin{tabular}{|c|c|c|c|c|}
\hline \multirow{2}{*}{ Variable } & \multicolumn{2}{|c|}{ Foveoschisis } & \multirow{2}{*}{ OR (CI 95\%) } & \multirow{2}{*}{ P-value } \\
\hline & Without & With & & \\
\hline \multicolumn{5}{|l|}{ Age (years) } \\
\hline$<45$ & $83(98.8 \%)$ & $1(1.2 \%)$ & OR 8.24 & \multirow[t]{2}{*}{$0.01^{\mathrm{a}}$} \\
\hline$\geq 45$ & $151(91.0 \%)$ & $15(9.0 \%)$ & CI 95\%: 1.05--62.77 & \\
\hline \multicolumn{5}{|l|}{$S E(D)$} \\
\hline$\geq-12 \mathrm{D}$ & $104(94.5 \%)$ & $6(4.5 \%)$ & & \multirow[t]{3}{*}{$0.00^{\mathrm{a}}$} \\
\hline$<-12$ to $-18 \mathrm{D}$ & $77(98.7 \%)$ & $1(1.3 \%)$ & & \\
\hline$<-18 \mathrm{D}$ & $53(85.4 \%)$ & $9(14.6 \%)$ & & \\
\hline \multicolumn{5}{|l|}{$P M$} \\
\hline No & $77(96.3 \%)$ & $3(3.7 \%)$ & OR 2.12 & \multirow[t]{2}{*}{$0.24^{\mathrm{a}}$} \\
\hline Yes & $157(92.4 \%)$ & $13(7.6 \%)$ & CI 95\%: $0.58-7.58$ & \\
\hline \multicolumn{5}{|l|}{$P C$} \\
\hline No & $126(97.6 \%)$ & $3(2.4 \%)$ & OR 5.05 & \multirow[t]{2}{*}{$0.00^{\mathrm{a}}$} \\
\hline Yes & $108(89.2 \%)$ & $13(10.8 \%)$ & CI 95\%: 1.39-18.01 & \\
\hline \multicolumn{5}{|l|}{$P R$} \\
\hline No & $195(96.5 \%)$ & $7(35 \%)$ & OR 6.42 & \multirow[t]{2}{*}{$0.00^{\mathrm{a}}$} \\
\hline Yes & $39(81.3 \%)$ & $9(17.3 \%)$ & CI 95\%: $2.24-18.20$ & \\
\hline \multicolumn{5}{|l|}{ Tractional structures } \\
\hline No & $223(97.3 \%)$ & $6(2.7 \%)$ & OR 33.78 & \multirow[t]{2}{*}{$0.00^{\mathrm{a}}$} \\
\hline Yes & $11(52.3 \%)$ & $10(47.7 \%)$ & CI 95\%: $10.34-109.39$ & \\
\hline \multicolumn{5}{|l|}{ Epiretinal membrane } \\
\hline No & $204(96.2 \%)$ & $8(3.8 \%)$ & OR 6.80 & \multirow[t]{2}{*}{$0.00^{\mathrm{a}}$} \\
\hline Yes & $30(78.9 \%)$ & $8(21.1 \%)$ & CI 95\%: $2.36-19.38$ & \\
\hline
\end{tabular}

Abbreviations: $\mathrm{CI}$, confidence intervals; $\mathrm{D}$, diopters; OR, odds ratio; PC, paravascular cyst; PM, paravascular microfold; PR,paravascular retinoschisis; $\mathrm{SE}$, spherical equivalent.

${ }^{\mathrm{a}} \chi^{2}$-test.

Table 3 Multivariate analysis for factors associated with myopic foveoschisis

\begin{tabular}{lccc}
\hline & OR & CI 95\% & P-value \\
\hline $\mathrm{SE}<-12 \mathrm{D}$ & 16.55 & $2.75-99.30$ & 0.00 \\
$\mathrm{PC}$ & 1.87 & $0.16-20.90$ & 0.61 \\
$\mathrm{PR}$ & 11.65 & $1.58-85.89$ & 0.01 \\
Tractional struct. & 17.76 & $3.45-91.33$ & 0.00 \\
ERM & 2.38 & $0.46-11.79$ & 0.30 \\
Age $>45$ years & 15.16 & $0.93-245.90$ & 0.06 \\
\hline
\end{tabular}

Abbreviations: CI, confidence intervals; ERM, epiretinal membrane; OR, odds ratio; PC, paravascular cyst; PR, paravascular retinoschisis; $\mathrm{SE}$, spherical equivalent.

have not identified any association between a longer AL and MF, even though, we observed that eyes with MF were longer than those without MF. This fact makes us consider the AL as an insufficient factor to develop MF on its own.

We speculate that AL might be a complementary force enhancing the action of the intraocular factors. Most cases of MF had a favorable evolution after vitrectormy with internal membrane peeling. However, there are some cases where MF persists and the development of foveal detachment and macular hole occurs after surgery. ${ }^{12,13}$ Wu $e t ~ a l^{5}$ claimed that this occurs because of the action of residual outer ocular forces. However, it can be because of different factors that are not eliminated with the surgery, such as the retina arteriolar elongation. ${ }^{7}$

In the multivariate analysis paravascular cysts, epiretinal membrane, and age were not significant. The factors associated with MF in the multivariate analysis are PR, tractional structures, and SE lower than -12D. This could be because paravascular cysts and retinoschisis are related to each other. Epiretinal membrane also disappear because it is included in the tractional structures. Age may disappear because we are studying progressive diseases, and all the variables studied are related to age. Myopic fundus changes are seldom found in children. ${ }^{14}$

In conclusion, we consider the PMs as the abnormality most often observed in the paravascular area in highly myopic eyes. MF would be a result of the action of different forces (intra- and extra-ocular forces), specially tractional structures, on precursor lesions (paravascular cyst and paravascular restinoschisis). The AL would be a complementary factor in the development of MF. Further studies with larger cases of MF are necessary to confirm these results due to the limitations of our study.

\section{Summary}

What was known before

- There different paravascular abnormalities in high myopia. Shimada reported that paravascular cysts were considered the most frequent one.

What this study adds

- We consider the paravascular microfolds as the paravascular abnormalities most often observed in highly myopic eyes. The presence of these abnormalities could precursor lesions of myopic foveoschisis.

\section{Conflict of interest}

The authors declare no conflict of interest.

\section{References}

1 Takano M, Kishi S. Foveal retinoschisis and retinal detachment in severely myopic eyes with posterior staphyloma. Am J Ophthalmol 1999; 128: 472-476.

2 Baba T, Ohno-Matsui K, Futagami S, Yoshida T, Yasuzumi K, Kojima A et al. Prevalence and characteristics of foveal retinal detachment without macular hole in high myopia. Am J Ophthalmol 2003; 135: 338-342.

3 Ikuno Y, Sayanagi K, Ohji M, Kamei M, Gomi F, Harino S et al. Vitrectomy and internal limiting membrane peeling for myopic foveoschisis. Am J Ophtalmol 2004; 137: 719-724.

4 Panozzo G, Mercanti A. Optical coherence tomography findings in myopic traction maculopathy. Arch Ophthalmol 2004; 122: 1455-1460.

5 Wu PC, Chen YH, Chen CH, Shin SJ, Tsui CL, Kuo KH. Factors associated with foveoschisis and foveal 
detachment without macular hole in high myopia. Eye 2009; 23: 356-361.

6 Shimada N, Ohno- Matsui K, Nishimuta A, Moriyama M, Yoshida T, Tokor T et al. Detection of paravascular lamellar holes and other paravascular abnormalities by optical coherence tomography in eyes with high myopia. Ophthalmology 2008; 115: 708-717.

7 Ikuno Y, Gomi F, Tano Y. Potent retinal arteriolar traction as a possible cause of myopic foveoschisis. Am J Ophthalmol 2005; 139: 462-467.

8 Sayanagi K, Morimoto Y, Ikuno Y, Tano Y. Spectral-domain optical coherence tomography findings in myopic foveoschisis. Retina 2010; 30: 623-628.

9 Ohno-Matsui K, Hayashi K, Tokoro T, Mochizuki M. Detection of paravascular retinal cysts before using OCT in a highly myopic patient. Graefes Arch Clin Exp Ophthalmol 2006; 244: 642-644
10 Sayanagi K, Ikuno Y, Gomi F, Tano Y. Retinal vascular microfolds in highly myopic eyes. Am J Ophthalmol 2005; 139: 658-663.

11 Soubrane G, Coscas GJ. La membrana neovascular coroidea en la miopía degenerativa. In: Ryan SJ, Hinton DR, Schachat, Wilkinson P (eds). Ryan Retina. Marban Libros: Madrid, Spain, 4 Ed., 2009, pp 1003-1020.

12 Hirakata A, Hida T. Vitrectomy for myopic posterior retinoschisis or foveal detachment. Jpn Ophthalmol 2006; 89: 1180-1183.

13 Sayanagi K, Ikuno Y, Takano Y. Reoperation for persistent myopic foveoschisis after primary vitrectomy. Am J Ophthalmol 2006; 141: 414-417.

14 Kobayashi K, Ohno-Matsui K, Kojima A, Shimada N, Yasuzumi K, Yoshida T et al. Fundus characteristics of high myopia in children. Jpn J Ophthalmol 2005; 49: 306-311. 